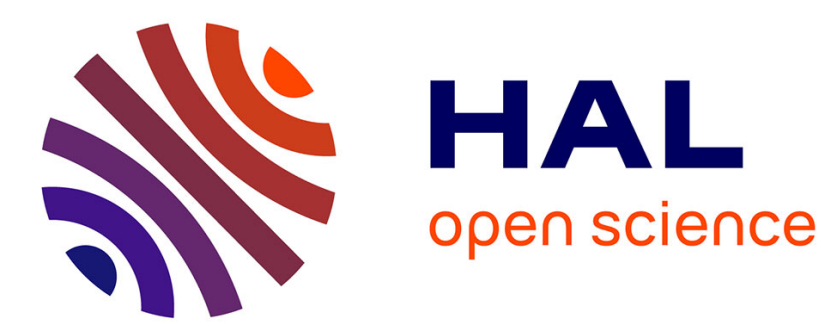

\title{
Oil repartition in a foam film architecture
}

Keyvan Piroird, Elise Lorenceau, Anne-Laure Biance

\section{To cite this version:}

Keyvan Piroird, Elise Lorenceau, Anne-Laure Biance. Oil repartition in a foam film architecture. Soft Matter, 2014, 10 (36), pp.7061. 10.1039/c4sm00538d . hal-01121910

\section{HAL Id: hal-01121910 https://hal-enpc.archives-ouvertes.fr/hal-01121910}

Submitted on 2 Mar 2015

HAL is a multi-disciplinary open access archive for the deposit and dissemination of scientific research documents, whether they are published or not. The documents may come from teaching and research institutions in France or abroad, or from public or private research centers.
L'archive ouverte pluridisciplinaire HAL, est destinée au dépôt et à la diffusion de documents scientifiques de niveau recherche, publiés ou non, émanant des établissements d'enseignement et de recherche français ou étrangers, des laboratoires publics ou privés. 


\title{
Oil repartition in a soap film architecture ${ }^{\dagger}$
}

\author{
Keyvan Piroird, ${ }^{a}$ Elise Lorenceau, ${ }^{b}$ and Anne-Laure Biance $* a$
}

\author{
Received Xth $X X X X X X X X X X 20 X X$, Accepted Xth $X X X X X X X X X 20 X X$ \\ First published on the web Xth $X X X X X X X X X X 200 X$ \\ DOI: 10.1039/b000000x
}

The propagation and distribution of oil inside the aqueous network of a foam is investigated in the case where oil can invade the foam without breaking it. The oil is injected into an elementary foam architecture of nine soap films and four vertices obtained after having plunged a cubic frame in a soap solution. The frame is then deformed to trigger a film switching (topological rearrangement named T1) and oil redistribution through this process is reported. Depending on the relative ratio of injected oil and water, different behaviours are observed. For small amounts of oil, a globule is trapped in one single node whereas for large oil volumes, it invades the four nodes of the soap film assembly. In both these cases, a T1 process does not change the oil distribution. However, for intermediate volumes, oil initially trapped in one node is able to propagate to the neighbouring nodes after the T1. This important observation shows that topological rearrangements, that naturally occur in foams when they evolve with time or when they flow, do affect the distribution of the third phase that they carry. These different regimes are captured by simple modeling based on capillary pressure balance inside the foam network. Moreover, in the large-oil-volume limit, a transient situation is evidenced where an oil film is trapped within the freshly formed water film. This oil film modifies the dynamics of the $\mathrm{T} 1$ process and can be stable up to a few minutes. We expect this mechanism to have consequences on the rheological properties of oil-laden foams. Film rupture dynamics is also experimentally captured.

\section{Introduction}

Liquid foams are dense assemblies of bubbles in a surfactant solution matrix. Due to their specific properties such as large specific area, low density or high thermal insulation, they are widely employed in industry as well as in everyday life (food industry, cosmetics, building materials, etc. $)^{1}$. They are also used to collect and selectively separate particulate materials in froth flotation, enhanced oil recovery or waste water decontamination $^{1,2}$. In these applications, liquid foams act as porous deformable media carrying a third particulate phase that may be either solid or liquid. In this context, we propose here to study the interplay of an aqueous liquid foam with oil globules.

When a foam is in contact with oil, different behaviours have been observed such as foam destabilisation ${ }^{3,4}$ or, on the contrary, foam stability enhancement ${ }^{5-10}$. These behaviours are intimately related to the structure of the surfactant micelles, the type of surfactant employed and the relative affinity of liquid, gas and oil ${ }^{11,12}$. This relative affinity is characterised by the stability of pseudoemulsion films, which are aqueous films intercalated at the oil/gas interface ${ }^{7,13-15}$. In particular, stable pseudoemulsion films allow to obtain stable oil-laden foams which are necessary in all extraction and decontamination processes. In that case, oil droplets trapped within the

† Electronic Supplementary Information (ESI) available: [details of any supplementary information available should be included here]. See DOI: $10.1039 / \mathrm{b} 000000 \mathrm{x} /$ foam network can deform the soft porous medium and even clog it as solid particles would do. This essentially limits the drainage and ultimately stabilises the foam ${ }^{5,10,16,17}$. Yet, unlike solid particles, large oil droplets are in turn deformed by the liquid network of the foam. Recent numerical ${ }^{18}$ and experimental ${ }^{19}$ studies have taken these deformations into account to determine the shape and the imbibition dynamics of an oil globule inside a single Plateau border.

We propose here to study oil repartition in a more complex soap film architecture made of multiple films, Plateau borders and nodes. We discuss oil repartition at equilibrium and emphasize its dynamical evolution under foam ageing. Indeed, foams are out-of-equilibrium systems, whose structure evolve with time due to drainage, coalescence, coarsening or flow ${ }^{20}$. Foam evolution is associated to elementary process in which bubbles exchange neighbours. This elementary process, during which the foam topology is modified, is called a T1 event. The dynamics of T1s is a subject of active research ${ }^{21-25}$ as it is a key ingredient in foam rheology ${ }^{26}$ and foam stability ${ }^{27}$. It is therefore crucial to determine how oil repartition is affected by $\mathrm{T} 1$ process and how the $\mathrm{T} 1$ dynamics is modified by the presence of oil. To answer that question, we investigate oil repartition after one single $\mathrm{T} 1$ triggered by modifying the architecture of an elementary foam consisted of a few soap films, in which visualisation is possible. 


\section{Experimental set-up}

The soap film architecture is generated on a cubic Plateau frame $^{28}$ with two sides of fixed length $(8 \mathrm{~mm})$ and one sliding side (fig. 1). (a)

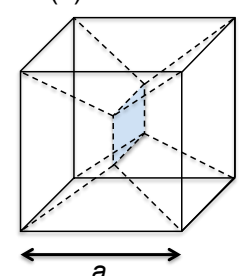

(b)

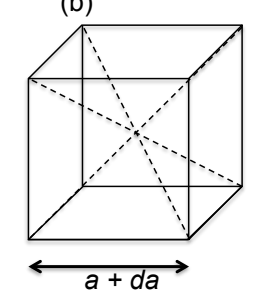

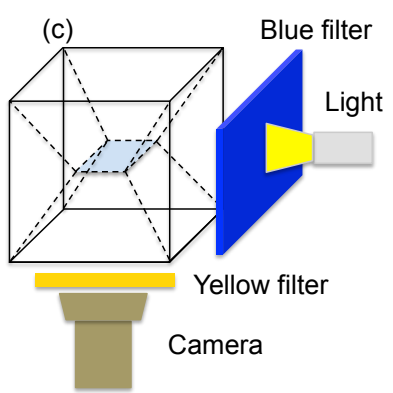

Fig. 1 Cubic frame of variable geometry used to form a minimal foam structure of nine soap films and to study an isolated T1 process. a) The frame is plunged into a foaming solution, resulting in the formation of a horizontal square soap film in the centre suspended to the frame by eight other films. b) By changing the length of the frame, the size of the central film is decreased until it totally disappears, leading to an unstable situation where eight Plateau borders meet at the same node. c) This triggers the T1 process and the opening of a new film perpendicular to the first one.

The frame is plunged into a foaming solution to form a central horizontal square film suspended by eight other soap films as in figure $1 \mathrm{~b}$. Following Plateau rules ${ }^{28}$, three films encounter at a liquid junction called Plateau border and four Plateau borders encounter in a vertex ${ }^{20}$. Our soap film architecture consists then of twelve Plateau borders (dashed lines of figure 1) and four nodes. The liquid fraction (or liquid pressure) of the film assembly is given by the radius of curvature of the Plateau borders $r_{p}$ defined in figure 2, which can be measured by image analysis with transmitted light. Once the film assembly is formed, $r_{p}$ is varied between $0.08 \mathrm{~mm}$ and 0.35 $\mathrm{mm}$ by drawing some liquid out with a tissue. The choice of the foaming solution and of the oil is limited to couples for which oil is able to enter the aqueous network keeping the soap film architecture stable. We mixed commercial dishwashing liquid ( $10 \%$ by weight, Manudish) with an equal fraction of glycerol to prepare the foaming solution. The air/solution surface tension is $\gamma_{w a}=28 \mathrm{mN} / \mathrm{m}$ (measured by the pendant drop method, Teclis). Sunflower oil (Carrefour) is employed for its relatively high surface tension in air: $\gamma_{o a}=32 \mathrm{mN} / \mathrm{m}$, which makes it prone to be covered by the foaming solution and thus facilitates the absorption by the foam. The interfacial tension between oil and the foaming solution is $\gamma_{o w}=0.6 \mathrm{mN} / \mathrm{m}$. Oil is withdrawn from a tank into a silica capillary tube of diameter $300 \mu \mathrm{m}$ connected to a syringe. The oil is then carefully injected in one of the upper Plateau borders by pushing it out of the capillary. We measure the length of the oil slug in the capillary before and after injection, which allows to define precisely the injected oil volume, which varies between 0.02 and
$1.5 \mu \mathrm{L}$. A fluorescent dye (fluorescent yellow 131SC, Dow, excitation wavelength: $494 \mathrm{~nm}$, emission wavelength: 535 $\mathrm{nm}$ ) is added to the oil to enhance the contrast with water. Oil repartition in the film assembly is observed via a camera (Marlin, $20 \mathrm{fps}$ ). The film assembly is lit with a LED, and is placed between a blue filter and a yellow collection filter in front of the camera objective. With this set-up, oil appears bright on the images and water is dark (except for a few reflections due to the curvature of the interfaces).

\section{Distribution of oil in the soap film architec- ture}

The distribution of a given volume $\Omega$ of oil in the aqueous network is represented in figure 2 as a function of the radius of curvature of the Plateau border $r_{p}$. Various behaviours are reported. For small oil volumes and large Plateau borders, the oil globule sits inside the first encountered node and remains almost spherical (fig. 2a). For intermediate $\Omega$ and $r_{p}$, the drop is strongly deformed in the node and partially spread along the neighbouring Plateau borders (fig. 2b). For large oil volumes and small nodes, the oil spreads further in the Plateau borders until reaching the neighbouring nodes. Thus, a continuous network of oil is observed through the node/Plateau border structure (fig. 2c).
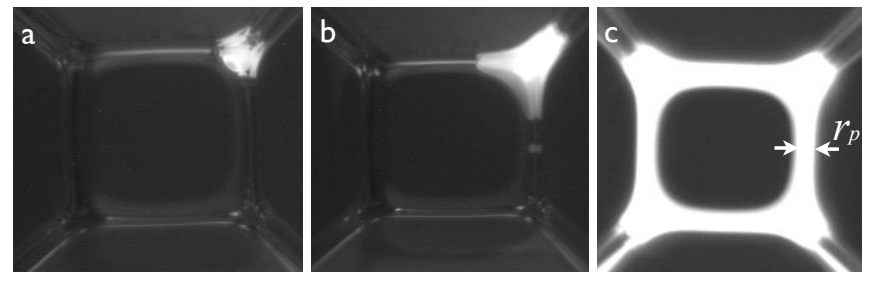

Fig. 2 Pictures taken from below in the configuration of figure 1c. The oil appears bright thanks to the fluorescent dye. a) Small oil volume in a large node: the oil drop is almost spherical and is trapped in the node. b) Intermediate oil volume and node: the oil is still in one node but also explores the neighbouring Plateau borders. The shape is not spherical anymore. c) Large oil volume in a small node: the oil invades the two neighbouring nodes and can even reach the fourth one as shown here. The distance between two nodes is $L_{n}=2 \mathrm{~mm}$ in all images.

Starting from these three situations, we trigger a T1 by reducing the length of the frame (transition from configuration of fig. 1c to fig. 1a). A new film perpendicular to the first one is created, only the upper two nodes being visible on the camera. Then, a second T1 is triggered by increasing the length of the frame back to its original position (transition from configuration of fig. 1a to fig. 1c). The small oil volumes (fig. 3a) are not affected by the topological rearrangement: they remain trapped in one node and oil repartition is unchanged before and after the T1s. For the intermediate situation (fig. 3b), the final state is different from the initial state: oil propagates 

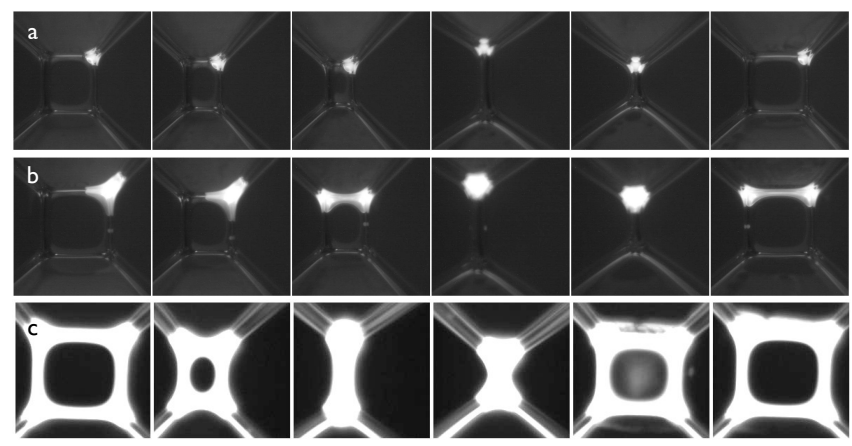

Fig. 3 Snapshots of oil repartition during a T1 process. Time interval is not constant between images. Starting from the three initial situations shown in fig. 2, a first T1 is triggered by reducing the length of the frame. This creates a new film that cannot be seen by the camera because it is orthogonal to the first one. We therefore trigger a second $\mathrm{T} 1$ by increasing the length of the frame back to its original position. a) Small oil volume. The drop remains trapped in one single node without affecting the T1 process. The first T1 occurs between frames 3 and 4 and the second between 5 and 6 . Initial and final states are identical. b) Intermediate oil volume. When the node containing the oil gets close to another empty node, oil is transferred and occupies two nodes and one Plateau border (frame 3 ). This happens just before the first $\mathrm{T} 1$, which occurs between frames 3 and 4 . The second $\mathrm{T} 1$ occurs between frames 5 and 6 and the final state is different from the initial state. c) Large oil volume. First T1 occurs between frames 2 and 3. After the second $\mathrm{T} 1$ between 4 and 5 , we observe a transitory situation where an oil film is trapped within the freshly created water film. After a certain time (varying between roughly one second and one minute), the oil film break and retracts to the Plateau borders while the water film is still here.

during the T1s when an oil-filled node merges with an empty one. We emphasize the following observations. First, the oil globule does not break during the rearrangements: in the final configuration, a slender oil slug confined in the Plateau border links the oil globules trapped in adjacent nodes. Moreover, the T1s-induced-transition is irreversible, the final configuration remains the same no matter how many $\mathrm{T} 1$ processes are performed. Finally, for large oil volumes, initial and final situations are again identical, but the system goes through a transitory state, in which an oil film gets trapped in the freshly created water film. This situation is discussed in more details in section 4 .

Occurrences of these behaviours are reported in the phase diagram of figure 4 as a function of $\Omega$ and $r_{p}$. Black dots correspond to the experiments illustrated in figures $2 \mathrm{a}$ and $3 \mathrm{a}$, where oil is trapped in one single node. Experiments where oil propagates to the neighbouring nodes after the $\mathrm{T} 1$ are marked with a grey dot. White dots represent experiments where oil spontaneously invades several nodes. In the following, we call $\Omega_{1}$ the critical volume for the transition between white and grey points and $\Omega_{2}$ for the grey/black transition. $\Omega_{1}$ and $\Omega_{2}$ are well defined and both increase with $r_{p}$.

To model the transition between these regimes, we evaluate the oil distribution between a node and the four adja-

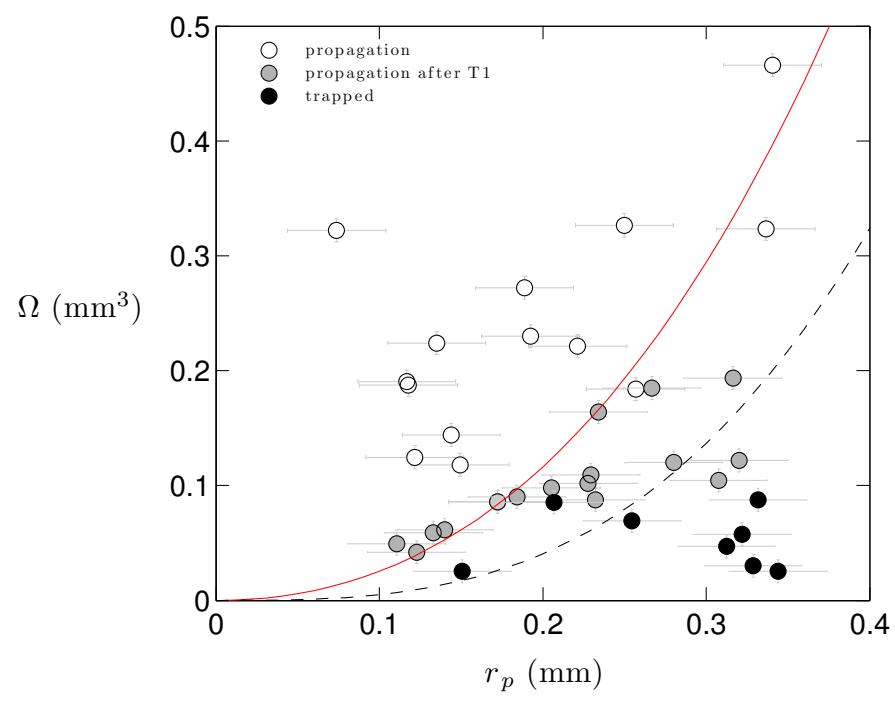

Fig. 4 Phase diagram showing oil repartition for various oil volumes $\Omega$ and Plateau border size $r_{p}$. Each point is an experiment. Black points correspond to situations where the volume of oil is small and trapped in one node, as in fig. 2a. White points represent experiments where the oil directly reaches the neighbouring nodes (fig. 2c) and grey points represent intermediate cases where the oil is initially deformed in one node (fig. $2 b$ ) and able to propagate to neighbouring nodes after a T1 (fig. 3b). Red solid line is $\Omega_{1}$ (eq. (3)) and dashed line represents $\Omega_{2}$ (eq. (4)).

cent Plateau borders. Since the Plateau border network is deformable, two conditions are required, which are volume conservation and pressure balance. As drawn in figure 5, we

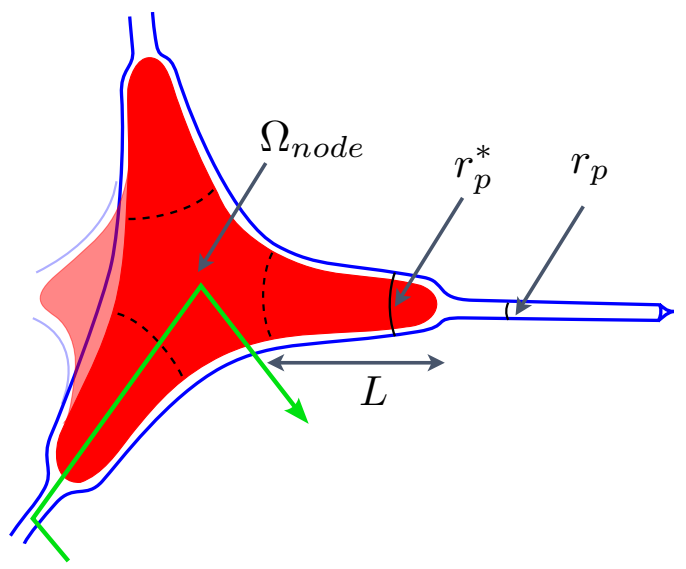

Fig. 5 Schematic representation of an oil drop trapped in a node and exploring the adjacent Plateau borders on a length $L . r_{p}$ is the radius of curvature of the undeformed Plateau border. $r_{p}^{*}$ is the radius of curvature of the Plateau border deformed by the presence of oil. The green arrow represents the path used to write the pressure balance (eq. 1).

consider that the oil is separated into five parts, one in the node and the other four in the adjacent Plateau borders, taking the form of slugs of length $L$. The total oil volume is 
$\Omega=\Omega_{\text {node }}+4 \alpha r_{p}^{* 2} L$, where $\alpha r_{p}^{* 2}$ is the cross-sectional area of a Plateau border deformed by the presence of oil, with a radius of curvature $r_{p}^{*}$ and $\alpha=\sqrt{3}-\pi / 2$. To determine the length slug $L$, volume conservation condition is applied, which required an estimation of the node volume. The geometrical boundary between a node and its adjacent Plateau border is difficult to establish and different models are reported in the literature ${ }^{20,29-32}$. Here, the node volume is simply estimated by using the volume of a tetrahedral void, i.e. the free volume at the tetrahedral junction of four spheres of radius $a$ in close contact. Note that this approximation does not modify significantly the obtained results. The volume of the node is therefore $\Omega_{\text {node }}=\Omega_{\text {tetra }}-4 \Omega_{\text {int }}$, where $\Omega_{\text {tetra }}=2 a^{3} \sqrt{2} / 3$ is the volume of the regular tetrahedron of edge $2 a$ defined by the four centres of the spheres. $\Omega_{\text {int }}$ is the volume of the intersection between the tetrahedron and one of the spheres, which reads $\Omega_{i n t}=2 a^{3}(\pi-3 \operatorname{Arctan}(\sqrt{(2)})) / 3$. The radius of the sphere $a$ is set by a pressure balance between the nodes, which have a curvature of $2 / a$ and the Plateau borders, which have a curvature of $1 / r_{p}{ }^{*}$, leading to $\Omega_{\text {node }} \simeq 1.6621 r_{p}^{* 3}$. In the following, we note $\zeta$ such as $\Omega_{\text {node }}=\zeta r_{p}^{* 3}$.

To determine how Plateau borders and nodes are deformed by the presence of oil, we now consider the pressure balance in the system along the line represented in green in figure 5. The line starts in air at ambient pressure, enters in a Plateau border by crossing a water-air interface, then enters in oil and finally comes back into air at ambient pressure by crossing both oil-water and water-air interfaces through one side of the node. Each interface crossing induces a pressure variation determined by interface curvature and surface tension. Since the line begins and starts in air, the sum of all pressures along this line is equal to zero. Hence:

$$
-\frac{\gamma_{\mathrm{wa}}}{r_{p}}+\frac{2 \gamma_{\mathrm{ow}}}{\beta r_{p}^{*}}+\frac{\gamma_{\mathrm{ow}}+\gamma_{\mathrm{wa}}}{r_{p}^{*}}=0
$$

The curvature at the end of the oil slug is assumed to be equal to $2 / \beta r_{p}^{*}$, with $\beta r_{p}^{*}=(2 / \sqrt{3}-1) r_{p}^{*}$ the radius of the circle inscribed in a Plateau border of radius of curvature $r_{p}^{*}$. This results in:

$$
\delta=\frac{r_{p}^{*}}{r_{p}}=\frac{(2+\beta) \gamma_{\mathrm{ow}}+\beta \gamma_{\mathrm{wa}}}{\beta \gamma_{\mathrm{wa}}}
$$

$\delta$ is equal to 1.3 with our experimental values of surface tension. Combining this equation with the volume conservation yields to the following expression relating the length of the slugs $L$, the volume of injected oil $\Omega$ and the radius of curvature of Plateau border $r_{p}: \Omega=\zeta \delta^{3} r_{p}^{3}+4 \alpha L \delta^{2} r_{p}{ }^{2}$. In our experimental configuration, the distance between two nodes $L_{n}$ is equal to $2 \mathrm{~mm}$. Using this value for $L$, we obtain the critical volume $\Omega_{1}$ that represents the transition between a drop that directly propagates to the neighboring nodes $\left(L>L_{n}\right.$, white points in fig. 4) and drops that remains trapped in a single node ( $L<L_{n}$, grey points).

$$
\Omega_{1}=\zeta \delta^{3} r_{p}^{3}+4 \alpha L_{n} \delta^{2} r_{p}^{2}
$$

Equation (3) is plotted as a red solid line in figure 4 without any adjustable parameter and accounts well for the transition that we observe experimentally.

To account for the second transition (between grey and black points in figure 4 ), we recall different general features concerning the geometry of the foam during the $\mathrm{T} 1$ process. The length of the Plateau borders vanishes from 2 to $0 \mathrm{~mm}$, to be swallowed by the nodes. The node volume remains however constant as the liquid has time in this quasi-static configuration to redistribute through adjacent Plateau borders. Then, the transition occurs only if oil is able to explore the second node, i.e. if the length of the slug $L$ is larger than the node size. T1 is triggered when the two nodes merge, so when the distance between their center is equal to their size $2 r_{p}^{*}$. The oil slug is therefore able to explore the empty node if i $L=r_{p}^{*}$. This suggests a critical volume $\Omega_{2}$ for the second transition of:

$$
\Omega_{2}=\delta^{3} r_{p}^{3}(\zeta+4 \alpha)
$$

This equation, represented by the blue dashed line in figure 4 without any adjustable parameter, is in correct agreement with experiments.

\section{Formation of an oil film during topological rearrangement}

As previously shown, the architecture of a minimal foam laden with oil globules exhibits a rich variety of structures which can be activated by T1 process. Another crucial point concerns the dynamics of these T1s in the presence of oil in the soap film architecture. The characteristic relaxation time of a $\mathrm{T} 1$ process is defined as the time it takes to go from the unstable situation illustrated in figure $1 \mathrm{~b}$ (where the four nodes coincide) to the final state where the new film is formed and at rest. For our solution and in the absence of oil, this time is on the order of one second. Measurements show that there is no significant deviation of the T1 dynamics when low and intermediate volumes of oil are injected.

However, if the four nodes and Plateau borders surrounding the central film are filled with oil (fig. 2c), the T1 dynamic is strongly affected. Triggering a T1 from this initial condition can lead to a transitory situation where an oil film is trapped within the freshly created water film, as seen in the 5th frame of figure 3c. Indeed, if the volume of oil is large enough, the generation of the foam film stretches out an oil film within it. The oil film slows down the T1 process and prevents the system to reach the equilibrium configuration given by the 
Plateau rules ${ }^{28}$. The system is stuck until the oil film ruptures, which occurs after a time $t_{f}$ comprised between a few seconds and one minute. The rupture of the oil film and its retraction into the Plateau borders is shown in figure 6 . We call $t_{r}$ the retraction time, between first and last frames of fig. 6, which is typically less than one second. Note that the break-up process does not alter the water film, which is remarkably stable (otherwise the whole foam architecture would collapse).

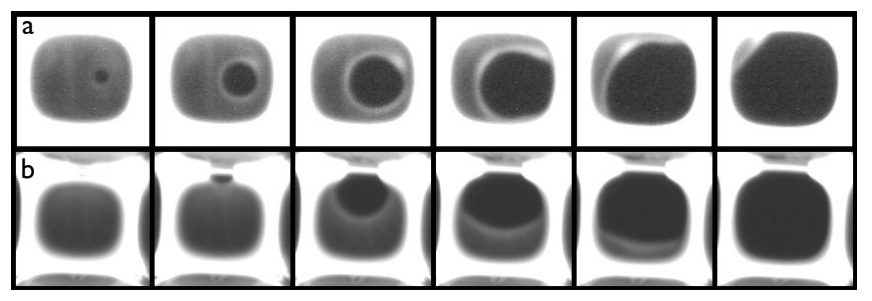

Fig. 6 Series of pictures showing how the oil film formed after a T1 breaks and retracts into the Plateau borders. Two distinct modes of rupture are observed. a) A hole nucleates in the oil film and grows until it reaches the Plateau borders. We clearly see a rim around the hole, that becomes thicker as the radius of the hole increases. The rim is not stable and its thickness is not uniform (starting from the third frame). This oil film lasted for $t_{f}=12 \mathrm{~s}$ before breaking. Interval between images: $167 \mathrm{~ms}$. b) For small oil volumes (typically below $0.5 \mu \mathrm{L}$ ), we observe another kind of rupture that is initiated from one of the Plateau borders. The oil comes off the top Plateau border on the second frame. The oil film is therefore unhooked and retracts. At the end, there is no oil in the film nor in the top Plateau border (The thin white line left on the top Plateau border is only a light reflection). This oil film lasted for $t_{f}=2 \mathrm{~s}$ before breaking. Interval between images: $125 \mathrm{~ms}$.

\subsection{Mechanisms of rupture}

Two modes of oil film rupture have been observed. For large oil volume, the film breaks by nucleation of a hole within the oil film (fig. 6a). For smaller volumes, the film unhooks from one of the edged Plateau borders (fig. 6b). We report in figure $8 \mathrm{a}$ the occurrence of these two behaviours as a function of the injected oil volume $\Omega$ for a constant value of $r_{p}=0.3 \mathrm{~mm}$. A critical volume $\Omega_{3}$ of roughly $0.6 \mu \mathrm{L}$ distinguishing these two behaviours can be defined. We also measure the life-time of the oil film $t_{f}$ (time between the T1 and the rupture). Oil films that rupture by unhooking from the Plateau border have short life-times, typically $t_{f}<4 \mathrm{~s}$ whereas films that rupture by hole nucleation last significantly longer as shown in figure $8 \mathrm{~b}$.

We make a quantitative use of the fluorescence signal to deduce the thickness of the oil film from the analysis of grey levels. The temporal evolution of the thickness in the centre of the film after the $\mathrm{T} 1$ is reported in figure 7 for the two cases shown in figure 6 . The thickness in the centre first decreases until it reaches a value of about $10 \mu \mathrm{m}$. This actually corresponds to the formation of a dimple of oil, that is clearly seen in the 5th image of figure $3 \mathrm{c}$. After a few seconds, this dimple drains out into the Plateau borders, leaving an homogeneous

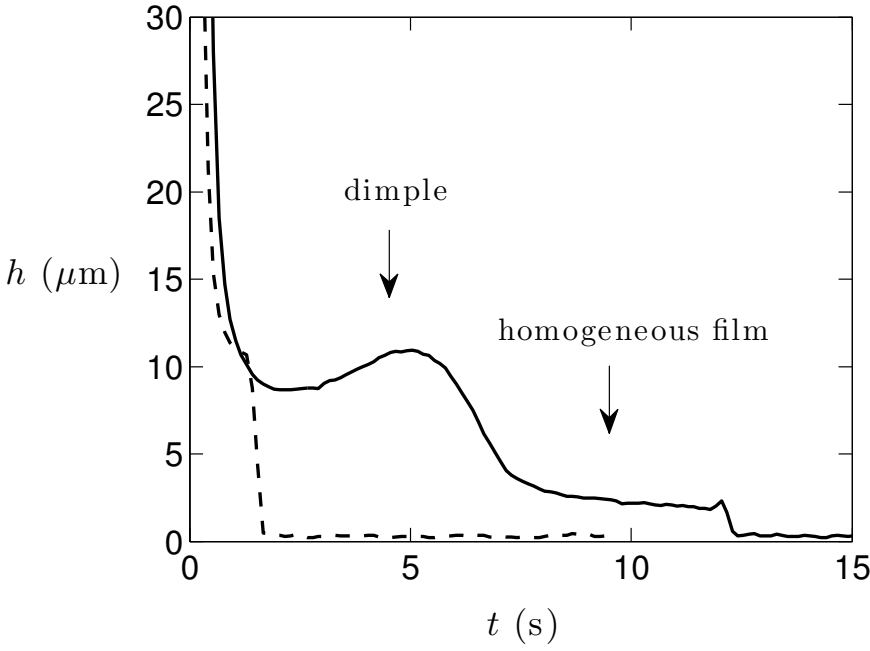

Fig. 7 Oil film thickness, measured at the centre of the film by grey level intensity, versus time. $t=0$ corresponds to the T1. The solid line corresponds to figure $6 \mathrm{a}$ (hole nucleation) and the dashed line to figure $6 \mathrm{~b}$ (rupture from the Plateau border).

oil film a few micrometer thick. Therefore, the rupture by hole nucleation always occurs where the film thickness is homogeneous whereas the unhooking from Plateau border always occurs when the dimple is still present, or even earlier, during the $\mathrm{T} 1$ process.

The value of the critical volume appearing in figure 8a, that marks the transition between the two modes of rupture must be equal to the volume of oil needed to fill the whole foam architecture, i.e. the four Plateau borders, the four nodes and the film. Indeed, for smaller oil volume, the oil reservoir is not sufficient and the film breaks before reaching its complete stretching. This volume is $\Omega_{3}=4 \alpha r_{p}^{* 2} L_{n}+4 \zeta r_{p}^{* 3}+h L_{n}^{2} \simeq$ $0.63 \mu \mathrm{L}$, for a film thickness $h=10 \mu \mathrm{m}$, a value that agrees
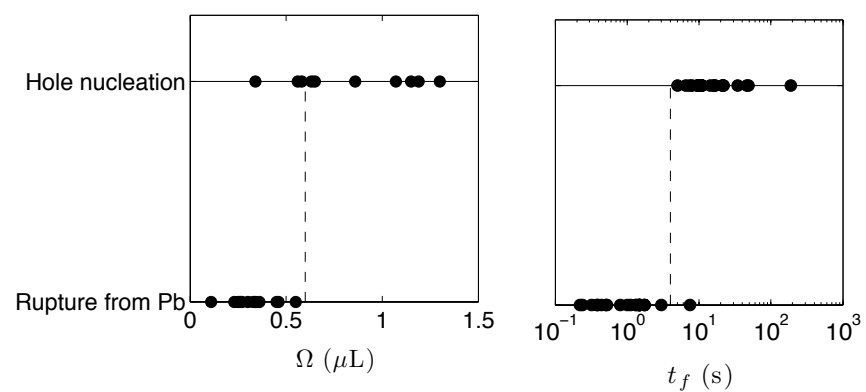

Fig. 8 Occurrence of rupture by hole nucleation (fig. 6a) and by rupture from the Plateau border (fig. 6b). a) Mode of rupture as a function of the volume of oil $\Omega$. b) Modes of rupture as a function of the time $t_{f}$ elapsed between the $\mathrm{T} 1$ and the rupture, corresponding to the life-time of the oil film. A critical volume $\left(\Omega_{3}=0.63 \mu \mathrm{L}\right)$ and a critical time $\left(t_{f}=4 \mathrm{~s}\right)$ are defined by the dashed lines on the figures. 
fairly well with our measurement (fig. 8a).

\subsection{Rupture dynamics}

When the film is trapped, the rupture dynamics is investigated. The variations of the radius of a hole in the oil trapped film versus time are reported in figure 9. The hole opens at a constant velocity, in good agreement with dynamics of soap film bursts in air as proposed by Taylor and Culick ${ }^{33,34}$. However, on the contrary to this situation, here the hole opening velocity, deduced from the ratio of $L_{n}$ with the film rupture duration $t_{r}$ increases with oil film thickness, as reported in the inset of figure 9. Bursting dynamics of a liquid soap film in a liquid environment is more complex ${ }^{35,36}$, and the viscous drag on the rim must be taken into account. In the case of a liquid film plunged in an infinite environment, an almost constant velocity is recovered, of the order $\gamma_{\text {ow }} / \eta_{\text {out }}, \eta_{\text {out }}$ being the viscosity of the external phase. However, in our experimental configuration where the environment is not infinite, the velocity depends on oil thickness and is largely below $\gamma_{\text {ow }} / \eta_{\text {out }}$, of the order of $1 \mathrm{~m} / \mathrm{s}$. Taking the viscosity of oil $\left(\eta_{\text {oil }}=50 \mathrm{mPa} . \mathrm{s}\right)$ instead of water gives $\gamma_{\mathrm{ow}} / \eta_{\text {oil }} \sim 1 \mathrm{~cm} / \mathrm{s}$, which is closer to what we observe but still higher and does not account for the dependence with $h$. This suggests that other modes of dissipation must be taken into account, such as surface energetic costs associated to the deformation of the water/air interface during film retraction due to the presence of a rim. This generates an excess of water/air interface, which has a huge energetic cost and then limits hole opening dynamics. A detailed analysis would require the exact shape of the water profile, as well as proper dissipation in the liquid. A precise modeling of bursting dynamics would necessitate to vary oil and water viscosity as their relative thickness, which is not in the scope of this work.

\section{Conclusions}

In a dedicated experiment mimicking an elementary foam, oil repartition in a soap film architecture has been experimentally investigated, when the oil is injected directly in a node. Depending on the volume of oil injected and the initial liquid content in our soap film assembly, different configurations have been observed. Large oil volumes injected in a dry foam are characterised by spontaneous imbibition, while smaller volumes remain confined at the injection point. Moreover, dynamical effects such as topological T1 transitions can induce propagation from one node to another. The T1 process then allows the oil globule to explore other configurations, which can be energetically favourable. The complexity of this problem is a consequence of the ability of large oil drops to deform, a feature different from what is observed in particle laden foam or emulsion laden foam, where the initial dimension of

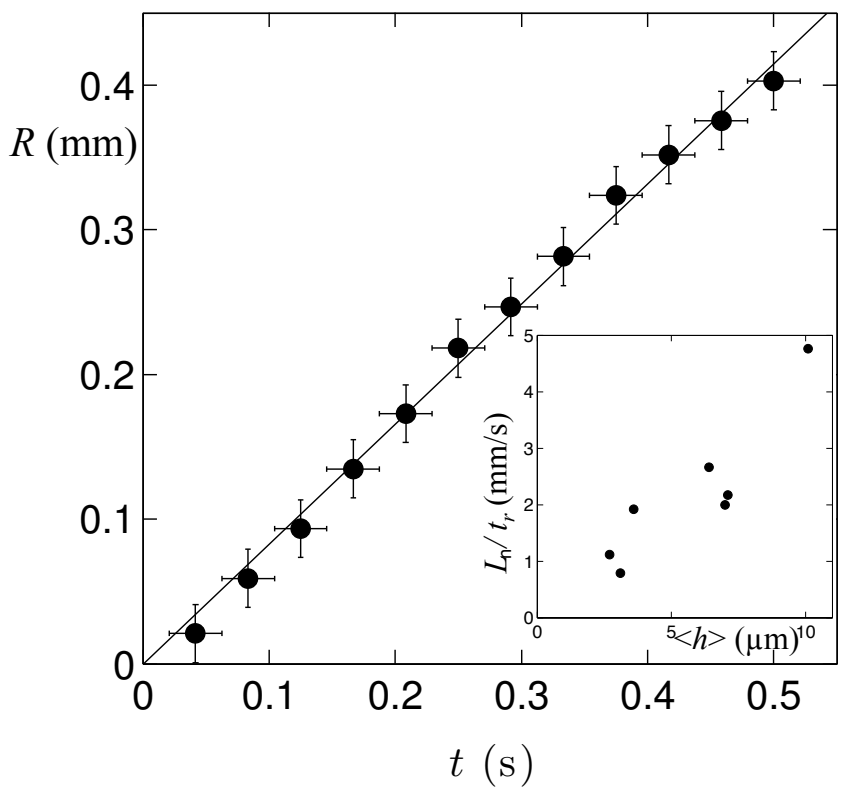

Fig. 9 Radius of the hole in the oil film as a function of time. $t=0$ corresponds to the instant when the hole appears. The measurement is made on the sequence corresponding to the first three frames in figure 6a. Inset: average opening velocity of the oil film, corresponding to the length $L$ of the film divided by the retraction time $t_{r}$, as a function of the film thickness $h$ (averaged over the whole film prior just before rupture).

the oil globule is so small that they appear as undeformable. We emphasize that such T1 induced oil propagation mechanisms and film trapping have been observed in macroscopic foams (see Supplementary materials). The oil distribution appears freezed after a $\mathrm{T} 1$ process, the propagation appearing irreversible. However, in real foam, the structure, and notably the size of the Plateau borders, evolve with time, due to gravity-driven drainage for example. We could therefore expect a variation of the transition thresholds between trapped and propagating oil globules, and thus a variation of the oil repartition with time. We have also shown that the presence of oil strongly affect T1 dynamics when an oil film is trapped within the freshly formed water film. These observations are a first step towards the comprehension of oil-laden foam rheology. A complete understanding of oil film rupture time and opening dynamics would be necessary to capture all the T1 dynamics in the presence of oil.

\section{Appendix: Supplementary materials}

Supplementary data associated with this article can be found, in the online version, at http://XXX. 


\section{Acknowledgements}

We thank Pauline Petit for experimental help and fruitful discussions and Remy Mensire for the supplementary materials. This project has been funded by Agence Nationale de la Recherche (ANR-11-JS09-012-WOLF) and by CNES through convention CNRS/CNES number 127233.

\section{References}

1 Foam Engineering: fundamentals and applications, ed. P. Stevenson, Wiley-Blackwell, 2012.

2 J. Rubio, M. L. Souza and R. W. Smith, Minerals Engineering, 2002, 15, 139-155.

3 P. Garrett, Defoaming: theory and industrial applications, CRC, 1992.

4 B. Binks and T. Horozov, Colloidal Particles at Liquid Interfaces, Cambridge University Press, 2012.

5 K. Koczo, L. Lobo and D. Wasan, J. Coll. Int. Sci., 1992, 150, 492-506.

6 R. Aveyard, B. Binks, P. Fletcher, T. Peck and P. Garrett, J. Chem. Soc. Faraday Trans., 1993, 89, 4313-4321.

7 V. Bergeron, M. Fagan and C. Radke, Langmuir, 1993, 9, 1704-1713.

8 E. Basheva, D. Ganchev, N. Denkov, K. Kasuga, N. Satoh and K. Tsujii, Langmuir, 2000, 16, 1000-1013.

9 E. Basheva, S. Stoyanov, N. Denkov, K. Kasuga, N. Satoh and K. Tsujii, Langmuir, 2001, 17, 969-979.

10 A. Salonen, R. Lhermerout, E. Rio, D. Langevin and A. Saint-Jalmes, Soft Matter, 2012, 8, 699-706.

11 J. Lee, A. Nikolov and D. Wasan, J. Coll. Int. Sci., 2014, 415, 18-25.

12 M. Simjoo, T. Rezaei, A. Andrianov and P. L. J. Zitha, Coll. Surf. A, 2013, 438, $148-158$.

13 L. Lobo and D. Wasan, Langmuir, 1993, 9, 1668-1677.

14 V. Bergeron, P. Cooper, C. Fischer, J. Giermanska-Kahn, D. Langevin and A. Pouchelon, Coll. Surf. A, 1997, 122, 103-120.

15 N. Denkov, Langmuir, 2004, 20, 9463-9505.

16 J. Goyon, F. Bertrand, O. Pitois and G. Ovarlez, Phys. Rev. Lett., 2010, 104, 128301.

17 N. Louvet, R. Höhler and O. Pitois, Physical Review E, 2010, 82, 041405.

18 S. J. Neethling, G. Morris and P. R. Garrett, Langmuir, 2011, 27, 97389747.

19 K. Piroird and E. Lorenceau, Physical Review Letters, 2013, 111, 234503.

20 I. Cantat, S. Cohen-Addad, F. Elias, F. Graner, R. Höhler, O. Pitois, F. Rouyer and A. Saint-Jalmes, Les Mousses. Structure et Dynamique., Belin (Echelles), Paris., 2010.

21 M. Durand and H. A. Stone, Phys. Rev. Lett., 2006, 97, 226101.

22 A.-L. Biance, S. Cohen-Addad and R. Höhler, Soft Matter, 2009, 5, 46724679.

23 A.-L. Biance, A. Calbry-Muzyka, R. Höhler and S. Cohen-Addad, Langmuir, 2012, 28, 111-117.

24 M. Le Merrer, S. Cohen-Addad and R. Höhler, Phys. Rev. Lett., 2012, 108, 188301.

25 M. Le Merrer, S. Cohen-Addad and R. Höhler, Phys. Rev. E, 2013, 88, 022303.

26 K. Martens, L. Bocquet and J.-L. Barrat, Soft Matter, 2012, 8, 4197-4205.

27 A.-L. Biance, A. Delbos and O. Pitois, Phys. Rev. Lett., 2011, 106, 068301-.

28 J. Plateau, Statique expérimentale et théorique des liquides soumis aux seules forces moléculaires, Gauthier-Villars, 1873.

29 N. Louvet, Ph.D. thesis, Université Paris-Est, 2009.

30 S. A. Koehler, S. Hilgenfeldt and H. A. Stone, Langmuir, 2000, 16, 63276341.
31 R. Phelan, D. Weaire, E. Peters and G. Verbist, Journal of Physics: Condensed Matter, 1996, 8, L475.

32 B. Haffner, Y. Khidas and O. Pitois, Soft Matter, 2014.

33 G. TAYLOR, Proceedings Of The Royal Society Of London Series AMathematical And Physical Sciences, 1959, 253, 313-\&.

34 F. E. C. Culick, Journal of Applied Physics, 1960, 31, 1128-1129.

35 P. Martin, A. Buguin and F. Brochard-Wyart, EPL (Europhysics Letters), 1994, 28, 421.

36 E. Reyssat and D. Quéré, EPL (Europhysics Letters), 2006, 76, 236. 